\title{
Combining Confocal and Other Optical Microscopies with Atomic Force Microscopy Imaging
}

J. Cleveland, I. Revenko, R. Proksch, Asylum Research, 6310 Hollister Ave., Santa Barbara, CA 93117.

Combining Atomic Force Microscopy (AFM) and confocal microscopy has long been of interest for biologists. These two types of microscopies individually provide unique information about samples. Researchers have turned to confocal microscopy for functional imaging of biological samples. Immunofluorescent labels can target molecules of interest with high specificity. With atomic force microscopy, a true, high resolution 3-D picture of the sample can be obtained at the nanoscale. Now researchers can learn much more about their samples by combining confocal microscopy with AFM imaging. With this combined techniques, researchers are able to:

1.) Optically locate features on the biological sample (Figure 1).

2.) Perform both AFM and confocal measurements (Figure 2). In addition, both confocal data and AFM data may be overlaid to yield additional information about the sample (Figure 3).

In this presentation, we'll discuss the instrumentation that is used for combined AFM and confocal microscopy. We'll also discuss specific application examples as well as other simultaneous AFM and optical microscopy techniques including epifluorescence, TIRF and phase contrast imaging. 


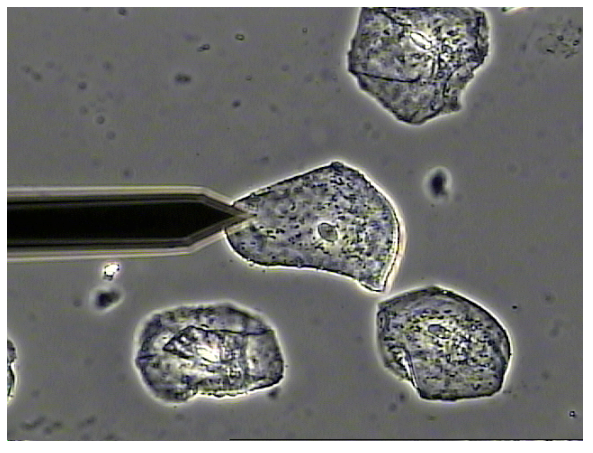

Fig. 1: Optical phase contrast imaging of epithelial cells on glass, also showing the AFM cantilever.

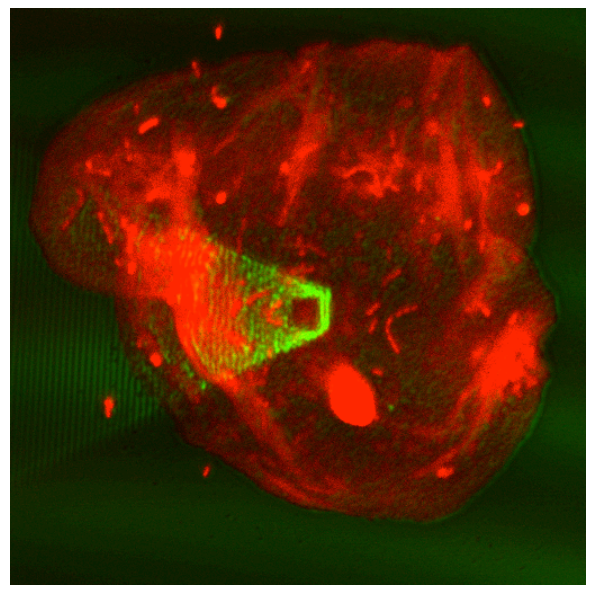

Fig. 2: Confocal image of cheek epithelial cell and bacteria labeled with ethidium bromide. The AFM cantilever is the bright green triangular feature.
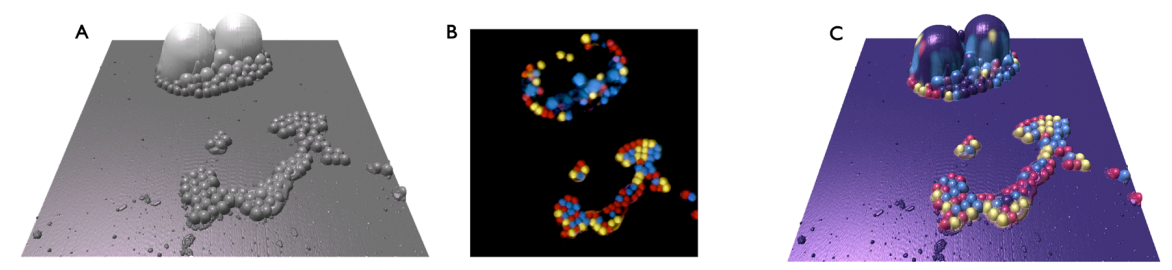

Fig. 3: Fig. 3A shows the AFM topography of fluorescent microspheres rendered in 3D, 35 $\mu \mathrm{m}$ scan. Fig. 3B shows the separate confocal data 505-600BP (blue), 530-600BP (green), 560LP (red). Excitation at $458 \mathrm{~nm}$ and $514 \mathrm{~nm}$. Fig. 3C shows the confocal data overlaid on the AFM rendered topography. 